\title{
Current Perspective on the Pathogenesis of Small Intestinal Neuroendocrine Tumors: Progress in Biomarkers and Molecular Events
}

\author{
Ying-Xuan Chen Jing-Yuan Fang \\ Division of Gastroenterology and Hepatology, Renji Hospital, Shanghai Jiaotong University \\ School of Medicine, Shanghai Institute of Digestive Disease, Shanghai, China
}

\section{Key Message}

A greater understanding of the molecular mechanisms that underpin the pathogenesis of small intestinal neuroendocrine tumors (SI-NETs) facilitates the classification, diagnosis and treatment of these relatively rare gastrointestinal malignancies.

\section{Practical Implications}

Currently, SI-NETs are diagnosed using histological examination and staining for various neuroendocrine markers. Genetically, SI-NETs are characterized by an absence of alterations to K-ras, p53 and DNA mismatch repair genes. Loss of chromosome 18, deletion of Smad2 and Smad4, and amplification of SRC, EGFR and PDGFR have been reported. Abnormal DNA methylation status, reflected by overexpression of DNA methyltransferase, higher methylation of the RASSF1A promoter and overexpression of histone $\mathrm{H} 1 \mathrm{x}$ are also associated with SI-NETs. These tumors are also associated with fibrosis, possibly due to the high levels of serotonin and other fibrotic factors produced. Genetic studies have pinpointed genes that can differentiate SI-NETs from other neuroendocrine tumors (oxytocin receptor, G protein-coupled receptor 113, VMAT-1, CDX-2), enabling more accurate diagnosis. Paraneoplastic antigen Ma2, neurokinin A and the CART peptide are under investigation as prognostic biomarkers. There is, however, still an unmet need for more sensitive biomarkers for earlier diagnosis and for a more specific classification system that encompasses tumor histology and reliable predictors of clinical response. 
Chen et al.: Current Perspective on the Pathogenesis of Small Intestinal

Neuroendocrine Tumors: Progress in Biomarkers and Molecular Events

\title{
Key Words
}

Biomolecular changes · Ileal carcinoid · Neuroendocrine tumors · Small intestinal · Tumor biomarkers

\begin{abstract}
Background: Neuroendocrine tumors of the gastrointestinal tract differ in their histopathologic and clinical presentation. Small intestinal neuroendocrine tumors (SI-NETs), representing only a small portion within gastrointestinal malignancies, are often associated with a delayed diagnosis due to their non-specific symptoms. The increased incidence of SI-NETs during the last decades demands earlier diagnosis and more effective treatment, which both rely on a better understanding on the underlying molecular mechanisms. Summary: The purpose of this review is to discuss the biomolecular changes responsible for the pathogenesis of SI-NETs, and potential biomarkers in the diagnostic and prognostic evaluation. @ $2013 \mathrm{~S}$. Karger AG, Basel
\end{abstract}

\section{Introduction}

Although the small bowel makes up approximately $80 \%$ of the total length and $90 \%$ of the absorptive surface of the alimentary tract, and despite the fact that its anatomic location is between two areas of high malignancy incidence, only $2 \%$ of all gastrointestinal tumors occur in the small intestine [1]. Small intestinal neuroendocrine tumors (SI-NETs), generally well-differentiated and having a more indolent clinical course, are the most frequent neoplasm encountered in the small bowel. Currently, their incidence has increased by more than 4 -fold (from 2.1 to 9.3 per million), according to the Surveillance, Epidemiology and End Results (SEER) database during 1973-2004 [2]. In addition, the ileum has been demonstrated to be the highest-risk site for SI-NETs, as it is affected about 7 times more frequently than the duodenum and the jejunum [3]. However, because of the vague symptoms, relatively rare incidence, low sensitivity and specificity of small intestine imaging techniques, the majority of SI-NETs are diagnosed at an advanced stage, while local or distant metastasis already having taken place, and consequently exhibit a poor prognosis. About $6-30 \%$ of patients with SI-NETs may present with carcinoid syndrome, which is usually associated with the development of liver metastases. This syndrome includes one or more of the following signs: flushing, diarrhea, abdominal discomfort, bronchial constriction and carcinoid heart disease. It occurs when hormones, mainly serotonin, tachykinins and prostaglandins, synthesized and secreted by the tumor, bypass the hepatic metabolism and enter the systemic circulation.

The optimal choice of treatment strategy depends on tumor size, histological features, cancer staging as well as the general health of the patient. Surgical resection is the basic treatment for patients without metastases or those with local complications of bowel obstruction or mesenteric ischemia. Due to the low proliferation rate of SI-NETs, conventional chemotherapeutics are not recommended for this disease. In terms of medical therapies, controlling symptoms by somatostatin analogs either alone or in combination with interferon still remains a priority. Although novel therapeutic approaches are under investigation, such as mammalian target of rapamycin inhibitors or peptide receptor radionuclide therapy, the incidence of SI-NETs continues to increase at an alarming pace, with minimal improvement in 5-year survival rates [4].

In this review article, we will summarize the recent insights into the genetic or epigenetic changes of SI-NETs, with specific focus on the current status of immunohistochemical and molecular markers in the diagnosis, prediction of tumor behavior and prognosis. 
Chen et al.: Current Perspective on the Pathogenesis of Small Intestinal

Neuroendocrine Tumors: Progress in Biomarkers and Molecular Events

\section{Nomenclature and Classification}

The appropriate nomenclature and classification of SI-NETs has long been a controversial topic. The variable classification systems that employ different terminology increase the difficulty in summarizing the information from previous literature.

Based on the tame appearance and less aggressive behavior compared to other tumor types of the same organ, 'carcinoid tumor' was the term initially proposed by Oberndorfer in 1907 [5] to characterize the submucosal lesions of the small intestine. In 1963, Williams and Sandler divided gastrointestinal NETs into three categories on the basis of their embryologic origin: foregut carcinoids (stomach, pancreas, duodenum, upper jejunum), midgut carcinoids (lower jejunum, ileum, appendix, cecum) and hindgut carcinoids (colon and rectum). In spite of the popular utility of these terms on a worldwide scale, it has become apparent that the word 'carcinoid' fails to reflect the real entity of these tumors, and so does the classification to separate the groups with distinctive prognosis [5].

Recently, the World Health Organization has revised the criteria for a classification system and divided them into (a) well-differentiated endocrine tumors (non-invasive tumors with features consistent with benign behavior or uncertain malignant potential), (b) welldifferentiated neuroendocrine carcinoma (presence of metastases or infiltration into the muscularis propria or angioinvasion), and (c) poorly differentiated neuroendocrine carcinoma (small cell carcinoma and large cell neuroendocrine carcinoma) [6], attempting to reach a consensus in these issues. However, benefiting from the rapid development of gene expression and molecular profiles of SI-NETs, it is likely that the above taxonomy could be modified to provide a more accurate definition of tumor subtypes.

\section{Biomolecular Changes Responsible for the Pathogenesis Process}

\section{Genetic Alterations Identified in SI-NET Disease}

SI-NETs are stable cancers with a lower mutation rate than that of breast, colorectal or lung cancers [7]. In SI-NETs, absence of K-ras mutations, deficiency in p53 alterations and DNA mismatch repair have been reported, which may account, at least in part, for their low proliferation activity.

On the other hand, several studies clarified that deletions of chromosome 18 were found in most of the SI-NET cases. However, exome sequencing of the candidate tumor suppressor genes in this region DCC, Smad4/DPC4 and Smad2/MADR2/JV18-1, revealed no mutation [8, 9]. The frequent loss of chromosome 18 implies that it may be the site of an as yet uncharacterized tumor suppressor gene involved in the early stage of tumorigenesis. Another study including 48 SI-NETs with integrative analysis of somatic single nucleotide variants and somatic copy number variations reported recurrently deletion of Smad2 and Smad4, as well as amplification of SRC, EGFR and PDGFR.

\section{Epigenetic Changes May Be Implicated in Malignant Transformation}

In addition to the inherited traits controlled by genes, epigenetic abnormalities may also play an important role in the malignant transformation of SI-NETs. Abnormal DNA methylation is regarded as one of the most common epigenetic changes observed in human cancers. Nuclear staining of DNA methyltransferase (DNMT) 1, 3a and 3b yielded a high frequency of DNMT overexpression $(87,81$ and $75 \%$, respectively) in gastroenteropancreatic NETs. Expression of DNMT1 did not differ according to anatomic sites, while DNMT3a and DNMT3b showed a significantly lower level in midgut than in foregut and hindgut NETs [10]. Epigenetic inactivation of Ras association domain-containing protein 1, isoform A (RASSF1A) is one of 
the most common molecular changes in cancer, associated with advanced tumor stage and poor prognosis. Similarly, higher methylation of RASSF1A promoter and lower expression of RASSF1A protein were discovered in metastatic tissue compared to primary small bowel carcinoid tumors [11].

Histone $\mathrm{H} 1 \mathrm{x}$, a subtype of the $\mathrm{H} 1$ histone family, is considerably expressed in tumor cells of SI-NETs when compared to corresponding normal tissues, regardless of the heterogeneous grade of their malignancy. Further investigation is required to clarify the underlying mechanism of this phenomenon and whether this $\mathrm{H} 1$ subtype participates in the tumorigenesis of NETs [12].

\section{Possible Mechanistic Explanations of Fibrosis Development}

A hallmark of SI-NETs is the notable fibrosis occurring either in the local mesentery, namely desmoplastic response, or distant organs, such as the heart. It is incurable with currently available therapeutics in the majority of patients. Based on the related literature, a potential mechanistic explanation has been proposed.

The large amount of serotonin produced by the neoplasm may be responsible for the fibrosis. Long-term administration of serotonin resulted in heart valve fibrosis in animal models, which resembles human carcinoid cardiac disease [13]. Other well-characterized fibrotic factors are the transforming growth factor- $\beta$ (TGF- $\beta$ ) family members, which have been found to be positively expressed in midgut carcinoids. As an example, overexpression of TGF- $\beta$ RII was particularly detected in stromal cells adjacent to the tumors [14], indicating its role in both cell-cell and cell-matrix interactions. As the downstream mediator of TGF- $\beta 1$, connective tissue growth factor (CTGF) functions in concert with TGF- $\beta 1$ in the stellate celldriven fibrotic response of SI-NETs [15]. A high level of CTGF is associated with cell migration, a reactive and well-vascularized myofibroblast-rich stroma and fibrosis development locally as well as in distal organs [16]. When blocking the serotonin receptor $2 \mathrm{~B}$ in a neoplastic EC cell line, KRJ-1, TGF- $\beta$ and CTGF levels were decreased, favoring the hypothesis that serotonin may act as the essential upstream factor in the development of fibrosis. Tryptophan hydroxylase 1 (TPH1), the initial step and rate-limiting enzyme involved in the synthesis process of serotonin, was found to be highly expressed in ileal carcinoid tissue [17]. Additionally, decreased expression of TPH1 and serotonin were observed [18], indicating a positive feedback serotonin autoregulation in SI-NET carcinogenesis.

Activation of the ERK pathway appears to be a common feature of SI-NETs, and the regulation of phosphorylated ERK1/ 2 was associated with the stimulation of serotonin and TGF- $\beta$ $[18,19]$. A recent study found that expression of CTGF was not induced by the TGF- $\beta$-mediated Smad signaling pathway but by TGF- $\beta$-activated ERK, JNK and p38 MAPK signaling in hepatic progenitor cells [20]. To our knowledge, no study has been carried out to confirm a similar mechanism existing in SI-NETs, which may offer novel attractive therapeutic targets.

\section{Biochemical Markers in the Diagnosis and Prognosis of SI-NETs}

\section{Traditional Histological and Serology Diagnostic Methods}

Currently, SI-NETs are diagnosed by histological examination and immunohistochemical staining for different neuroendocrine markers. In a study including 167 jejunoileal carcinoid cases, the positive rates by immunohistochemistry were 98, 96, 95, 92 and 86\% for argentaffin, argyrophil, neuron-specific enolase, chromogranin and serotonin, respectively [21]. Measurement of 24-hour urinary 5-hydroxyindoleacetic acid and serum chromogranin A (CgA) levels also serves as a method to confirm the diagnosis and monitor tumor burden and recurrence [22]. Recently circulating pancreastatin, a fragment of the CgA molecule, was considered as a new sensitive marker for disease progression [23]. 
Chen et al.: Current Perspective on the Pathogenesis of Small Intestinal

\section{Potential Biomarkers for Differential Diagnosis}

Growing evidence indicates the difference in genetics, biology and response to treatment between pancreatic neuroendocrine tumors and SI-NETs, despite their continued inclusion and analysis as a homogeneous group in some articles. DNA microarray analysis validated the significant difference in gene expression between these tumor types [24], allowing for a differential diagnosis depending on gene expression profile. Furthermore, another study using $G$ protein-coupled receptor arrays identified two genes (oxytocin receptor and G proteincoupled receptor 113) that were upregulated by more than five-fold in all SI-NET samples, while no pancreatic neuroendocrine tumors shared this profile [25]. Vesicular monoamine transporters (VMATs) facilitate the transport of monoamines from the cytoplasm into the secretory vesicles of neurons and neuroendocrine cells. Overexpression of VMAT-1 mRNA and protein level was described in primary as well as metastasized ileal carcinoids $[17,26]$, and statistically significant upregulation of the VMAT-1 gene (22-fold) in SI-NETs was also observed in one study mentioned above [25]. Moreover, the mutually exclusive prevalence of VMAT-1 in serotonin-containing cells and of VMAT-2 in histamine-secreting cells could enable pathologists to distinguish SI-NETs among other types of NETs. CDX-2, a homeobox gene encoding a nuclear transcription factor that is essential for intestinal cell differentiation and development, was proved to be highly specific for ileal NETs and their metastatic tumors [27, 28].

A panel of these different diagnostic biomarkers may improve the prediction of the primary tumor. A decision tree supplemented with a pruning algorithm was proposed by Modlin et al. [29] and used to diagnose SI-NETs based on the assessment of circulating markers transcript levels (TPH1, CgA, DDC, neuron-specific enolase, VMAT-1 and VMAT-2). The sensitivity and specificity were reported to be 81.2 and $100 \%$, respectively.

\section{Biomarkers for Prognosis}

Paraneoplastic antigen Ma2 is displayed in SI-NET tissue [30]. In a study including 124 SI-NET patients at different stages of disease, measurement of Ma2 autoantibodies in blood samples showed a high sensitivity and specificity in discriminating SI-NET patients from the healthy as compared to the evaluation of serum CgA level, and those with a higher level of serum Ma2 autoantibodies may suffer a worse outcome [31]. Additionally, further exploration of the underlying mechanisms may provide novel insights into the potential autoimmunity-involved tumorigenesis of SI-NETs.

Serial determination of neurokinin A levels may be useful for the early identification of SI-NETs with a more aggressive behavior. Increasing neurokinin A level ( $>50 \mathrm{pg} / \mathrm{ml})$ is associated with a poor short-term survival, a need for immediate treatment, and also acts as a sensitive tool to monitor the effectiveness of therapy [32].

Cocaine- and amphetamine-regulated transcript (CART) peptide exerts several regulatory functions as a neurotransmitter or hormone. Landerholm et al. [33] identified the correlation between increasing CART score and worse disease-specific survival when adjusting for age, disease stage and tumor grade.

Due to the limited sample size of most research, those studies including diverse tumor types with a large number of cases are warranted to confirm the clinical relevance of a variety of biomarkers in the future.

\section{Conclusions}

Because of their rare incidence, the pathogenesis of SI-NETs remains largely unknown. Recent advances in molecular mechanism underlying the development of SI-NETs may improve the management of these tumors. In this review, evaluation of a variety of immuno- 
histochemical stainings, bimolecular alterations and pathologic findings was performed. There is still an unmet requirement for more sensitive and specific biomarkers for earlier diagnosis, as well as a more sophisticated classification system including both histological patterns and reliable molecular markers allowing prediction of therapy response and tumor behavior. Another challenge is the unclear therapeutic targets in clinical practice. Considering more than one mechanism implicated in these malignancies, the establishment of individualized multiple target interventions based on molecular alterations will be of major clinical significance, with the potential to enhance antitumor effects and to reduce side effects.

\section{References}

1 Neugut AI, Jacobson JS, Suh S, Mukherjee R, Arber N: The epidemiology of cancer of the small bowel. Cancer Epidemiol Biomarkers Prev 1998; 7:243-251.

2 Gustafsson BI, Siddique L, Chan A, Dong M, Drozdov I, et al: Uncommon cancers of the small intestine, appendix and colon: an analysis of SEER 1973-2004, and current diagnosis and therapy. Int J Oncol 2008;33:11211131.

3 Schimmack S, Svejda B, Lawrence B, Kidd M, Modlin IM: The diversity and commonalities of gastroenteropancreatic neuroendocrine tumors. Langenbecks Arch Surg 2011;396:273-298.

-4 Modlin IM, Champaneria MC, Chan AKC, Kidd M: A three-decade analysis of 3,911 small intestinal neuroendocrine tumors: the rapid pace of no progress. Am J Gastroenterol 2007;102:1464-1473.

5 Soga J: The term 'carcinoid' is a misnomer: the evidence based on local invasion. J Exp Clin Cancer Res 2009; 28:15.

6 Klimstra DS, Modlin IR, Coppola D, Lloyd RV, Suster S: The pathologic classification of neuroendocrine tumors: a review of nomenclature, grading, and staging systems. Pancreas 2010;39:707-712.

7 Banck MS, Kanwar R, Kulkarni AA, Boora GK, Metge F, et al: The genomic landscape of small intestine neuroendocrine tumors. J Clin Invest 2013;123:2502-2508.

8 Wang GG, Yao JC, Worah S, White JA, Luna R, et al: Comparison of genetic alterations in neuroendocrine tumors: frequent loss of chromosome 18 in ileal carcinoid tumors. Mod Pathol 2005;18:1079-1087.

-9 Lollgen RM, Hessman O, Szabo E, Westin G, Akerstrom G: Chromosome 18 deletions are common events in classical midgut carcinoid tumors. Int J Cancer 2001;92:812-815.

10 Rahman MM, Qian ZR, Wang EL, Yoshimoto K, Nakasono M, et al: DNA methyltransferases 1, 3a, and 3b overexpression and clinical significance in gastroenteropancreatic neuroendocrine tumors. Hum Pathol 2010;41: 1069-1078.

11 Zhang HY, Rumilla KM, Jin L, Nakamura N, Stilling GA, et al: Association of DNA methylation and epigenetic inactivation of RASSF1A and beta-catenin with metastasis in small bowel carcinoid tumors. Endocrine 2006; 30:299-306.

12 Warneboldt J, Haller F, Horstmann O, Danner BC, Fuzesi L, et al: Histone H1x is highly expressed in human neuroendocrine cells and tumours. BMC Cancer 2008;8:388.

13 Gustafsson BI, Tommeras K, Nordrum I, et al: Long-term serotonin administration induces heart valve disease in rats. Circulation 2005;111:1517-1522.

14 Chaudhry A, Funa K, Oberg K: Expression of growth factor peptides and their receptors in neuroendocrine tumors of the digestive system. Acta Oncol 1993;32:107-114.

15 Kidd M, Modlin I, Shapiro M, Camp R, Mane S, et al: CTGF, intestinal stellate cells and carcinoid fibrogenesis. World J Gastroenterol 2007;13:5208-5216.

16 Jacobson A, Cunningham JL: Connective tissue growth factor in tumor pathogenesis. Fibrogenesis Tissue Repair 2012;5(suppl 1):S8.

17 Vikman S, Essand M, Cunningham JL, de la Torre M, Oberg K, et al: Gene expression in midgut carcinoid tumors: potential targets for immunotherapy. Acta Oncol 2005;44:32-40.

18 Svejda B, Kidd M, Giovinazzo F, Eltawil K, Gustafsson BI, et al: The 5-HT(2B) receptor plays a key regulatory role in both neuroendocrine tumor cell proliferation and the modulation of the fibroblast component of the neoplastic microenvironment. Cancer 2010;116:2902-2912.

19 Kidd M, Modlin IM, Pfragner R, Eick GN, Champaneria MC, et al: Small bowel carcinoid (enterochromaffin cell) neoplasia exhibits transforming growth factor-beta1-mediated regulatory abnormalities including up-regulation of C-Myc and MTA1. Cancer 2007;109:2420-2431.

20 Ding ZY, Jin GN, Liang HF, Wang W, Chen WX, et al: Transforming growth factor beta induces expression of connective tissue growth factor in hepatic progenitor cells through Smad independent signaling. Cell Signal 2013;25:1981-1992.

21 Burke AP, Thomas RM, Elsayed AM, Sobin LH: Carcinoids of the jejunum and ileum. Cancer 1997;79:10861093. 
22 Modlin IM, Kidd M, Latich I, Zikusoka MN, Shapiro MD: Current status of gastrointestinal carcinoids. Gastroenterology 2005;128:1717-1751.

-23 O’Dorisio TM, Krutzik SR, Woltering EA, Lindholm E, Joseph S, et al: Development of a highly sensitive and specific carboxy-terminal human pancreastatin assay to monitor neuroendocrine tumor behavior. Pancreas 2010;39:611-616.

-24 Duerr EM, Mizukami Y, Ng A, Xavier RJ, Kikuchi H, et al: Defining molecular classifications and targets in gastroenteropancreatic neuroendocrine tumors through DNA microarray analysis. Endocr Relat Cancer 2008; 15:243-256.

25 Carr JC, Boese EA, Spanheimer PM, Dahdaleh FS, Martin M, et al: Differentiation of small bowel and pancreatic neuroendocrine tumors by gene-expression profiling. Surgery 2012;152:998-1007.

26 Voland P, Besig S, Rad R, Braun T, Baur DM, et al: Correlation of matrix metalloproteinases and tissue inhibitors of matrix metalloproteinase expression in ileal carcinoids, lymph nodes and liver metastasis with prognosis and survival. Neuroendocrinology 2009;89:66-78.

27 Jaffee IM, Rahmani M, Singhal MG, Younes M: Expression of the intestinal transcription factor CDX2 in carcinoid tumors is a marker of midgut origin. Arch Pathol Lab Med 2006;130:1522-1526.

28 Srivastava A, Hornick JL: Immunohistochemical staining for CDX-2, PDX-1, NESP-55, and TTF-1 can help distinguish gastrointestinal carcinoid tumors from pancreatic endocrine and pulmonary carcinoid tumors. Am J Surg Pathol 2009;33:626-632.

-29 Modlin IM, Gustafsson BI, Drozdov I, Nadler B, Pfragner R, et al: Principal component analysis, hierarchical clustering, and decision tree assessment of plasma mRNA and hormone levels as an early detection strategy for small intestinal neuroendocrine (carcinoid) tumors. Ann Surg Oncol 2009;16:487-498.

-30 Leja J, Essaghir A, Essand M, Wester K, Oberg K, et al: Novel markers for enterochromaffin cells and gastrointestinal neuroendocrine carcinomas. Mod Pathol 2009;22:261-272.

31 Cui T, Hurtig M, Elgue G, Li SC, Veronesi G, et al: Paraneoplastic antigen Ma2 autoantibodies as specific blood biomarkers for detection of early recurrence of small intestine neuroendocrine tumors. PLoS One 2010; 5:e16010.

-32 Diebold AE, Boudreaux JP, Wang YZ, Anthony LB, Uhlhorn AP, et al: Neurokinin A levels predict survival in patients with stage IV well differentiated small bowel neuroendocrine neoplasms. Surgery 2012;152:11721176.

33 Landerholm K, Shcherbina L, Falkmer SE, Jarhult J, Wierup N: Expression of cocaine- and amphetamine-regulated transcript is associated with worse survival in small bowel carcinoid tumors. Clin Cancer Res 2012;18: 3668-3676. 